Écrire

l'histoire

\section{Écrire l'histoire}

Histoire, Littérature, Esthétique

13-14 | 2014

Archives

\title{
Les registres du tribunal sharî̀ de Tripoli et du tribunal shar'î de Halbâ
}

Leur importance dans l'écriture de l'histoire du Liban

\section{Fârûq Hablas}

Traducteur : Amin Elias et Dominique Avon

\section{(2) OpenEdition}

Édition électronique

URL : http://journals.openedition.org/elh/479

DOI : $10.4000 /$ elh.479

ISSN : 2492-7457

Éditeur

CNRS Éditions

\section{Édition imprimée}

Date de publication : 10 octobre 2014

Pagination : 109-117

ISBN : 978-2-271-08208-4

ISSN : $1967-7499$

\section{Référence électronique}

Fârûq Hablas, "Les registres du tribunal sharî̀ de Tripoli et du tribunal sharî de Halbâ », Écrire l'histoire [En ligne], 13-14 | 2014, mis en ligne le 10 octobre 2017, consulté le 23 septembre 2020. URL : http:// journals.openedition.org/elh/479; DOI : https://doi.org/10.4000/elh.479 


\section{Les registres du tribunal shar'î de Tripoli et du tribunal shar'î de Halbâ}

\section{Leur importance dans l'écriture de l'histoire du Liban}

Extraits présentés et traduits de l'arabe par Amin Elias et Dominique Avon

Écrire l'histoire du Liban est une entreprise qui n'a, à ce jour, jamais abouti au sens où l'entend un lecteur européen'. Dans les accords de Taëf, signés par une majorité de parlementaires libanais en 1989 afin de mettre un terme à près de quinze années de guerres, figure une recommandation qui porte précisément sur la rédaction d'un manuel commun pour tous les Libanais. Les responsables politiques avaient conscience du fait que la confrontation des mémoires des communautés confessionnelles, plus encore que celle des classes sociales, empêchait toute constitution d'un tissu national, ultime garant de la stabilité du fragile État. L'ouvrage a vu le jour vingt ans plus tard, pour être immédiatement retiré de la circulation. L'histoire de la commission et des acteurs investis de cette mission académique a donné lieu à une thèse en science politique². L'article dont les extraits les plus significatifs ont été traduits dans les pages qui suivent, s'inscrit dans la première phase de ce processus inachevé.

L'historien présente le contenu de deux sources documentaires issues des tribunaux shar' $\hat{i}^{3}$ de Tripoli et de Halbâ. Il montre que, du fait même de la nature de l'institution, l'intérêt de ces archives se situe à tous les niveaux de l'histoire sociétale: juridique, social (jusqu'aux détails de la vie quotidienne), économique, religieux et politique. Son approche, ce qui est dit et ce qui est tu, n'est pas moins digne d'enseignement. Fârûq Hablas évoque la question de la localisation des archives, mais il ne s'interroge pas sur la conservation, la gestion et le libre accès aux archives. Il n'effleure pas les enjeux des controverses entre les historiographies de langue arabe et de langue turque, ceux-ciétant pourtant déjà connus des spécialistes4. II ne s'interroge pas sur les concepts utilisés, à commencer par celui 
de hukm appliqué à l'autorité exercée aussi bien par les Égyptiens que par les Ottomans, mais qui ne dit rien de la nature de ce régime. Il a été traduit par «dominance » pour rester au plus près de l'ambiguïté laissée par l'auteur. Mais le lecteur doit être conscient qu'il existe, dans les années 2000, des intellectuels du Caire, de Beyrouth ou de Damas, qui entendent appliquer le terme de «colonisation» à cette manière de pouvoir imposé par les vainqueurs turcs à partir $d u$ xvle siècle.

Avec des moyens modestes, les traducteurs de cet article ont tenté d'attirer l'attention de responsables, au sein du monde arabe, sur l'état problématique de la recherche et de l'enseignement de l'histoire. Il existe encore une très belle école historiographique libanaise, polyglotte, ayant reçu une formation de haut niveau. Mais ses membres sont vieillissants et la relève des générations est tout sauf assurées. Voici le "cri d'alarme» lancé en 2004 dans le pays, qui dispose sans doute des meilleures institutions universitaires de la région, à l'exception d'Israël: "Dans trois des principales Universités du Liban il n'y a plus aucun étudiant inscrit en formation initiale d'Histoire. À l'Université Libanaise, les effectifs ont décru de manière catastrophique en quelques années. Si la tendance se poursuivait, ne permettant pas le remplacement de générations bien formées, les conséquences pourraient être graves 6 .» Près d'une décennie plus tard, loin de s'être améliorée, la situation s'est encore dégradée en dépit d'initiatives ponctuelles', d'actions de mise en valeur du patrimoine intellectuel ${ }^{8}$ ou d'entreprises pédagogiques de

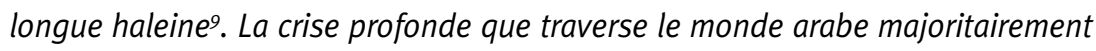
musulman, crise qui concerne le cadre étatique (à commencer par le tracé des frontières) comme la nature des régimes, ne favorise pas le renversement de tendance. Il importe que les lecteurs d'Écrire l'histoire en aient conscience.

\section{Notes}

1 Kamal SAlibi, Une maison aux nombreuses demeures. L'identité libanaise dans le creuset de l'histoire, trad. par Sylvie Besse Ricord, Naufal, 1989. Cet essai, initialement publié en anglais, a donné lieu à de nombreux débats entre historiens libanais: $c f$. Jean SHARAF, "Fî qirâ'a li-kitâb "Bayt bi manâzil kathîra". Lubnân bayn al-ta'rîkh al-îdyûlûjî̀ wa al-manhaj al-wad'î» [Lecture du livre Une maison aux nombreuses demeures. Le Liban entre l'historicité idéologique et la méthodologie positive], Majallat al-difấ' al-wațan $\hat{\imath}$, no 3,1990 , p. 165-190.

2 Betty Gilbert-Sleiman, Unifier l'enseignement de l'histoire dans le Liban d'après-guerre: conditions et limites de l'élaboration de la nouvelle politique publique du manuel scolaire d'histoire (1989-2001), thèse sous la direction d'Anțân Masarrah et Elizabeth Picard, Aix-Marseille 3 en cotutelle avec l'Université libanaise, 2010.

3 Tribunaux dans lesquels le droit islamique est appliqué en fonction des différentes écoles juridiques et du juge qui s'y réfère.

4 Stéphane Yerasimos, «Arabes et Turcs, un malentendu héréditaire», Historiens et géographes, n 336, mai-juin 1992, p. 295-304.

5 Parmi les historiens récemment décédés ayant joué un rôle majeur dans cette génération et travaillé dans ce champ de recherche, on retiendra le nom de Jean SHARAF, Jabal Lubnân fî al-qarn al-sâdis 'ashar. Al-Dîmughrâfyâ wa al-iqtisâd min khilâl al-dafâtir al-'uthmâniyya, Kaslik (Liban), PUSEK, 2008. 
6 La Nahda. Réveils de la pensé en langue arabe. Approches. Perspectives, actes du colloque organisé à l'USEK les 28-29 octobre 2004, Kaslik (Liban), PUSEK, 2009, p. 239.

7 Colloque sur l'enseignement de l'histoire organisé à l'USEK par le P. Karam Rizk.

8 Exposition «Cénacle libanais»; cf. Les Années Cénacle (1946-1975) entre histoire, mémoire et actualité, Beyrouth, Publications de la Fondation du Cénacle libanais, 2012.

9 Cours trilingues (français, anglais, arabe) sur l'histoire euro-méditerranéenne, réalisés par les universités de Genève, d'Agadir, de Kaslik et du Maine (Le Mans), <http://hemed.univ-lemans.fr/> .

Les archives ottomanes officielles ${ }^{1}$ constituent une source majeure pour étudierl'histoire du Liban etdu Bilâdal-Shâm² en général, durant la période de la dominance ottomane sur ces pays, entre 1516 et 1918. Ces archives ne se limitent pas seulement à celles qui dépendent du bureau du Vizir à Istanbul: une grande partie se trouve dans les départements locaux officiels créés par les Ottomans dans les vilayets d'Acre, de Tripoli, de Damas, d'Alep, etc. [Il faut] prendre en considération le fait que l'extension ou la multiplicité de ces départements dans le cadre de chaque vilayet est liée au changement des règlements administratifs ottomans $[\ldots]$, notamment durant la seconde moitié du xIX ${ }^{e}$ siècle. Durant l'époque des Tanzimat ${ }^{3}$, un tribunal shar $\hat{\imath} \hat{l}$, un département de jurisprudence, un conseil administratif, un département des registres des impôts et un département financier ont été créés au sein de chaque casa ${ }^{4}$. En outre, des tribunaux d'ordre général ont été créés au cœur des vilayets. Tous ces départements nous ont laissé leurs archives, comme les registres des départements financiers des vilayets et des casas et les registres de fiscalité foncière imposée à chaque village.

Ces diverses archives départementales, notamment celles des tribunaux shar $\hat{\imath} \hat{\imath}$, nous offrent une base de données historiques précise et détaillée, équiva- lente aux informations disponibles dans les archives d'Istanbul. Alors que dans ces archives les documents concernant nos contrées sont axés sur les notables, les adjudicataires, les recensements des habitants des villages ainsi que les impôts récoltés, les archives des tribunaux shar $\hat{i}$ comprennent des documents [plus précis]: les contrats de vente et de louage ainsi que les documents relatifs au statut personnel. Ces documents sont indispensables à l'écriture de l'histoire sociale et de l'histoire des relations socio-économiques. Cela ne veut pas dire que les archives [...] de ces départements peuvent se substituer aux archives d'Istanbul, mais que les deux dépôts se complètent. L'un et l'autre sont indispensables pour comprendre parfaitement notre histoire présente. Ils nous aident à saisir la réalité de notre société dans le passé ainsi que son évolution à travers son histoire contemporaine.

Mais avant d'aborder notre sujet par le détail, il importe de signaler que l'enregistrement de ces documents ne visait pas à aider les historiens, mais uniquement à conserver les dossiers au sein de l'État ottoman, notamment ceux qui concernaient la direction des vilayets et des régions, le contrôle et la rentrée des impôts ainsi que l'enregistrement des formalités relatives aux habitants afin de garantir les droits. 


\section{Les registres du tribunal shariî de Tripoli}

\section{A. - Leur nombre}

[...] Les archives du tribunal shar'î de Tripoli ${ }^{5}$ relevant de la période ottomane comptent 104 registres numérotés de 1 jusqu'à 119 et deux registres qui ne sont pas numérotés [...]. Les numéros que portent ces registres montrent que certains d'entre eux sont perdus. [p. 200]

\section{B. - La période couverte par les registres}

[...] Ces registres couvrent, dans leur ensemble, la période allant de 1077 (de l'hégire)/1666 jusqu'à 1339 (de l'hégire)/1920, laissant quelques vides. Ils couvrent aussi bien les périodes de la dominance ottomane que les périodes de la dominance égyptienne sur le Bilâd al-Shâm, comme les registres $\mathrm{n}^{\text {os }} 51,53$, $54,55,56$ et 59. [p. 212]

\section{C. - Le volume des registres}

[...] Le volume des divers registres diffère tant par le nombre de pages de chacun d'eux que par les dimensions de ces pages. Le nombre de pages varie de 135 à 600 pour chaque registre. Néanmoins, la plupart des registres comprennent plus de 400 pages.

S'agissant des dimensions, il existe des registres dont les dimensions sont de $45 \times 16 \mathrm{~cm}$, comme le registre $\mathrm{n}^{\circ} 8$. Il existe aussi des registres dont les dimensions sont de $37 \times 29 \mathrm{~cm}$, comme le registre $\mathrm{n}^{\circ} 40$, et d'autres dont les dimensions sont de $31 \times 21 \mathrm{~cm}$, comme le registre $\mathrm{n}^{\circ} 55$. Nous pouvons dire que la plupart des registres relevant des $\mathrm{XVII}^{\mathrm{e}}$ et $\mathrm{XIX}^{\mathrm{e}}$ siècles sont de grand format alors que la plupart de ceux qui proviennent de la fin du XVIII $^{\mathrm{e}}$ et du début du XIX ${ }^{\mathrm{e}}$ siècle sont de petit format. [p. 213]

\section{D. - La langue et le style}

La plupart des documents sont écrits en langue arabe. Certains, notamment les firmans, sont écrits en langue turque, suivis d'une traduction arabe. Quant aux documents comptables des gouverneurs, ils sont rédigés en langue turque sans traduction vers l'arabe. Nous observons aussi que les correspondances officielles sont rédigées en langue turque uniquement, dès le début du $\mathrm{xx}^{\mathrm{e}}$ siècle.

Les ordres édictés par les gouverneurs locaux ou de province sont, pour la plupart, rédigés en langue arabe, même ceux qui relèvent du $x^{e}$ siècle. [p. 215]

\section{E. - L'objet des documents}

Les registres $\mathrm{du}$ tribunal shar' $\hat{\imath}$ de Tripoli sont riches et diversifiés. Ils englobent tous les aspects de la vie quotidienne et des activités officielles du vilayet de Tripoli. [Ces documents] sont classés sous les titres suivants: titres de propriété; affaires ; firmans ; ordres ; notifications ou rapports; quittances.

Parmi ces registres figurent des documents relatifs au statut personnel des musulmans comme les certificats de mariage ou de divorce et aux frais afférents, les attestations de procuration, de pupillarité et de dévolution successorale. Des documents concernent les procès 
menés par des musulmans et des dhim$m i-\mathrm{s}^{6}$ et les affaires de discordes entre chrétiens et juifs. Figurent aussi des certificats relatifs à la libération d'esclaves, à des questions de vente ou de louage de terrains [...], de maisons et de produits commerciaux, de waqf $-\mathrm{s}^{7}$ et de filiation pour musulmans et dhimmi-s. De plus, il existe des certificats pour désigner des responsables et des comptables pour la gestion et la location des waqf-s. Il y a également des certificats pour désigner des écrivains publics de langue arabe et turque au tribunal shar $\hat{\imath}$, des muftis, des shérifs, des magistrats shar' $\hat{\imath}$, des prédicateurs dans les mosquées, des imams, des muezzins, des enseignants et des servants dans les mosquées et les écoles religieuses, ainsi que des certificats désignant des évêques et des patriarches pour les chrétiens.

Il y a aussi des listes de noms de places, de rues, de portes et de khâns de Tripoli, des noms de cheikhs de quartiers, ainsi que des impôts exigés des khâns.

[...] De même, il existe des listes d'impôts exigés de tous les dhimmi-s de Tripoli [...] ainsi que de chacun des monastères. Il y a, enfin, des certificats pour édifier des églises ou pour restituer d'anciennes églises ou des bâtiments publics (comme la forteresse de Tripoli et les bourgs du port). [p. 218-219]

\section{L’importance des archives du tribunal shar'î de Tripoli}

Les documents relatifs aux questions de divorce, de mariage, d'héritage et de vente de logements et aux frais associés nous donnent une idée claire de la vie de [chaque] famille, de son niveau social, de ses capacités matérielles et de ses relations sociales. Nous trouvons, dans les documents concernant le mariage, le montant des dots offertes aux femmes mariées et parfois les détails des trousseaux des jeunes épouses. Dans les documents relatifs aux questions d'héritage, des précisions concernent le legs du défunt, y compris ses vêtements, ses meubles, ses provisions de nourriture ainsi que [les noms] de ses enfants et de ses épouses. Nous trouvons aussi des détails concernant la description globale des maisons vendues ainsi que leur valeur foncière et leur situation géographique dans des actes de vente. Il y a autant d'indications sur le niveau social du propriétaire de la maison que sur ses biens matériels.

Quant aux documents relatifs aux questions de douane et de ventes de nourriture et de terrains, ils nous informent des prix du moment, du mouvement de vente des biens fonciers et de la tendance économique au cours d'une année précise. Les certificats concernant les adjudications des biens apportent des indications sur les professions et le règlement intérieur au sein des corporations professionnelles.

Les documents liés aux questions des adjudications de régions nous renseignent sur les divisions administratives dans le vilayet de Tripoli ainsi que sur les divisions intérieures propres à chaque région. Nous trouvons aussi, dans ces documents, les noms des adjudicataires et de leurs garants ainsi que les noms des villages et des cheikhs qui dépendent 
d'eux. En outre, ces documents nous donnent des détails sur les impôts exigés de ces villages, sur les compétences des adjudicataires et sur les devoirs de ces derniers envers le gouverneur. D'autres documents, relatifs à la sécurisation des régions et aux garanties proposées par les cheikhs des villages envers l'adjudicataire, révèlent une partie des relations entre ce dernier et les habitants. Ils témoignent aussi de la multiplicité des adjudicataires au cours d'une seule année et de la manière dont ils sont remplacés d'une année sur l'autre. Cela met en évidence l'intensité de la concurrence entre les notables pour diriger et gérer les régions.

[D'autres informations] se trouvent dans les documents liés aux opérations de vente des terrains agricoles, parmi lesquels un classement de ces terrains. Il s'agit de propriétés [privées], de propriétés du prince [i.e. le sultan] et de waqf-s. Ces documents nous révèlent aussi les diverses espèces de plantations, le montant des impôts annuels et des détails relatifs aux devoirs du locataire ou du paysan qui exploite le terrain pour le compte du propriétaire. Nous trouvons aussi des détails concernant le droit de celui qui travaille la terre et sa part sur la production dudit terrain. Cela nous aide à connaître le fondement et les différentes formes de la relation entre celui qui travaille la terre et le propriétaire.

Les documents concernant la désignation des notables ou des shérifs, des muftis, des prédicateurs, des imams, des enseignants dans les mosquées et des chargés des waqf-s révèlent des détails de la vie religieuse et des familles religieuses. Ils nous informent aussi sur les facteurs qui ont aidé à l'affirmation de l'autorité et à l'accumulation de richesses de certaines familles. Des postes religieux comme celui de mufti, par exemple, se transmettaient de génération en génération par héritage, il en allait de même pour le contrôle du waqf ou des institutions religieuses [...]. [p. 226-227]

\section{Les registres du tribunal shariî de Halbâs}

Il n'existait pas de tribunal shar' $\hat{\imath}$ à Halbâ avant la seconde moitié du $\mathrm{XIX}^{\mathrm{e}}$ siècle. Les formalités officielles des habitants du 'Akkâr' étaient enregistrées dans les registres du tribunal shar'î de Tripoli. C'est durant l'époque des Tanzimat que l'État ottoman a créé des tribunaux shar'î dans tous les casas, y compris celui du 'Akkâr. Le document le plus ancien attribué à un juge shar'î du 'Akkar remonte à 1266 (de l'hégire) / 1850. [...] Mais le feu qui a frappé le tribunal shar' $\hat{\imath}$ de Halbâ en 1958 a détruit quelques-uns de ses registres, n'en laissant que cinq, qui couvrent la période allant de 1303 (de l’hégire) / 1885 à 1340 (de l'hégire)/1921. Quant aux registres relatifs à la période 1266-1302 (de l’hégire)/1850-1884, ils sont tous détruits. De nos jours, il n'en reste que des copies conformes conservées dans les bibliothèques de certains habitants du 'Akkâr. Nous observons, à travers ces copies conformes, que le siège du tribunal shar'î du 'Akkâr a été déplacé du village de Birqâyil vers celui de Burj avant d'être établi à Halbâ.

Avant de parler des autres registres, nous attirons l'attention des chercheurs 
sur le fait que l'un des cinq registres est maintenant conservé aux archives du département $\mathrm{du}$ waqf islamique du
'Akkâr, alors que les quatre autres sont conservés dans le bâtiment du tribunal shar'î de Halbâ. [p. 235-236]

\section{L'importance historique des registres du tribunal shar'î de Halbâ}

Les documents relevant de ces registres nous présentent beaucoup de détails historiques. Ils ressemblent, tant par les détails qu'ils nous offrent à propos de tel ou tel événement que par leurs circonstances, à ceux du tribunal shar'î de Tripoli. Ces documents nous offrent une base de données historiques riche et diversifiée concernant tous les villages du 'Akkâr. Ils se révèlent très utiles dans l'écriture de l'histoire administrative et politique de cette région, car il n'existe pas de documents relatifs à la région du 'Akkâr dans [les archives] de l'administration ottomane. Ils concernent les chrétiens autant que les musulmans et nous informent sur le positionnement de chacune des communautés religieuses dans cette région à la fin $d u X x^{e}$ et au début du $x^{e}$ siècle. Ces documents nous permettent, grâce aux contrats de louage des terrains agricoles, de saisir la réalité des rapports entre le propriétaire et le paysan. La compa- raison de ces contrats avec ceux qui figurent dans les registres du tribunal shar î de Tripoli met en lumière le fait que les rapports ont évolué au profit des paysans. En outre, les contrats de vente des terrains agricoles enregistrés dans les registres du tribunal shar î de Halbâ nous aident à nous rendre compte du fait que certains notables ont vendu leurs terrains et en conséquence ont perdu le facteur principal sur lequel s'était bâtie leur notabilité. La majorité de ces documents nous renseigne sur l'apparition du leadership de quelques familles chrétiennes dans le 'Akkâr, et cela en raison de l'acquisition d'une fortune foncière considérable à la fin du XIX siècle par les chefs de ces familles. De plus, les documents relatifs aux questions de divorce et de mariage nous renseignent sur les relations sociales entre les habitants du 'Akkâr et leur voisinage durant cette période. [p. 240]

\section{Conclusion}

Il nous paraît évident que les archives des tribunaux shar î de Tripoli et de Halbâ constituent un trésor précieux et une mémoire fidèle des détails d'anciens événements quotidiens. Ces archives aident le chercheur et l'historien à comprendre la réalité historique des régions qui s'étendent du sud de Byblos jusqu'à la frontière nord [du Liban d'aujourd'hui] et du sommet des montagnes jusqu'à la 
côte. Pour cette raison, il est impossible de les ignorer quand on écrit l'histoire de ces régions. Plus encore, toute étude qui ne prendrait pas en compte ces archives serait imparfaite.

Ces archives qui ont été récemment découvertes, comme celles des tribunaux shar'î de Saïda, de Damas et de Beyrouth, tout autant que celles des monastères, des évêchés et des patriarcats, ouvrent la question de la nécessité de réécrire objectivement et par les documents l'histoire du Liban. Cette entreprise nous révélera la vérité de notre histoire et nous aidera à nous découvrir et à comprendre l'évolution de notre société.

Ce dont notre société d'aujourd'hui a le plus besoin, c'est de connaître la vérité historique. Il y a, dans les manières de penser et les interrogations des personnes cultivées, une envie de saisir la vérité historique en même temps que des doutes vis-à-vis de l'exactitude des [informations qu'elles reçoivent] et de la sincérité de ceux [qui leur pré- sentent l'histoire]. Elles ont raison [d'être méfiantes], eu égard aux contradictions qu'elles ont constatées dans les livres $\mathrm{d}^{\prime}$ histoire du Liban traitant et expliquant tel phénomène ou tel événement. Par conséquent, ces personnes cultivées se sont affolées et ont perdu leur confiance à la fois dans l'histoire et dans les historiens. Ce qui les a poussées à renier leur patrimoine et leurs aïeux. Et si tel est le cas des personnes cultivées, que dire des foules, prisonnières des histoires non documentées, vivant dans leurs rets et cherchant à bâtir la société sur leurs illusions?

Il est temps que les historiens libanais s'épaulent afin d'écrire une histoire unifiée de leur patrie à partir des seuls documents, une histoire qui ne vise que la mise au jour de la vérité. La vérité historique est un fondement indispensable pour consolider la société et la patrie et pour bâtir un avenir sûr pour les nouvelles générations.

\section{Notes}

1 L'original de ce texte de Fârûq Hablas, membre du département d'histoire de l'Université libanaise, a paru, sous le titre «Sijillât al-mahkama al-shar'îyya fî Tarâblus wa sijillât al-mahkama al-shar'îyya fî Halbâ wa ahammiyyatuhâ fî kitâbat târîkh Lubnân", dans la revue Dirâsât [«Études»] (Beyrouth, Faculté de l'éducation à l'Université libanaise), t. 1, nº 26 (spécial), 1997, p. 197-252. Nous remercions l'éditeur de nous avoir aimablement autorisés à en publier la traduction [NDLR].

2 Expression ambiguë qui recouvre, à la date de la rédaction de l'article, ce qui correspond à la Syrie, à la Jordanie, au Liban (qui se trouve donc deux fois englobé), à Israël et aux territoires palestiniens occupés. [Toutes les notes sont des traducteurs.]
3 Période de réformes institutionnelles et juridiques décidées au XIX ${ }^{\mathrm{e}}$ siècle par les autorités ottomanes sous la pression des puissances européennes.

4 Le casa est une circonscription administrative dans l'Empire ottoman.

5 Ville située au Nord-Liban, à 70 kilomètres de Beyrouth.

6 Terme désignant ceux qui ne sont pas musulmans (chrétiens et juifs dans ce cas), vivant selon un statut d'infériorité légale et fiscale et bénéficiant d'une protection officielle.

7 Biens de mainmorte selon le droit islamique. 
8 Ville située au Nord-Liban à 120 kilomètres de Beyrouth.

9 Région au nord du Liban; elle se situe sur la zone frontalière entre le Liban et la Syrie au moment où l'article est rédigé; $c f$. <http:// www.localiban.org/spip.php?article655>, cons. 4 oct. 2013. 